\title{
Design on Data Storage Structure for Course Management System
}

\author{
Li Ma \\ School of Education and Sports, Bohai University, Jinzhou, 121013, China \\ 88228471@qq.com
}

Keywords: course management system; data; storage structure; design; conceptual structure; logical structure

\begin{abstract}
Curriculum management is an important function of educational administration system. The traditional curriculum management has many disadvantages. It is difficult to meet the needs of the information age. In this paper, based on the Sybase database management system design, to lay the foundation for the rapid development of course management system. First, the structure of Sybase database system is studied, and the characteristics of Sybase database are explained. Secondly, the concept structure design, which shows the entity and the relationship between them by E-R diagram; Then, the logic structure design is logical structure of data table representation by way of the form; finally, storage and optimization design point out the storage space allocation method. The result of this study has an important role on improve the quality and efficiency of software development.
\end{abstract}

\section{Introduction}

Education is the fundamental source of the survival and development of our country. Through the cultivation of the needs of economic and social development of labor and for the society to create scientific knowledge and material wealth, promote economic growth, promote the prosperity of the nation and to promote the development, promote world peace and development of human beings. The education information has an important role on the development of education. In the work of teaching management in Colleges and universities, course management is a very complex task. With the popularization of higher education in the country, the number of colleges and universities is increasing. Course management is still using manual methods, which increases the difficulty of management and academic staff workload and hence it is difficult to meet the needs of the information age [1,2]. With the systematization and network of the teaching management in Colleges and universities, the course management system has become an important part of the teaching management. Development of course management system, can make the tedious teaching aids work become simple and easy, can also realize information standardization, science statistics and rapid inquiry, alleviate the burden of the staff, improve the efficiency of management. For the difficulty of system development, this paper based on the Sybase database management system for data storage structure design.

\section{Sybase Database System Structure}

Sybase database is a relational database system developed by American Sybase company. It is a large database system which is a typical Unix or WindowsNT platform client / server environment. Sybase provides a set of application programming interfaces and libraries that can be integrated with non-Sybase data sources and servers, allowing data to be replicated across multiple databases. System has a complete trigger, stored procedures, rules and integrity of the definition, to support the optimization of the query, with good data security.

Sybase database has the following characteristics [3]: programmable database, through the provision of stored procedures to create. Stored procedures allow users to write their own database subroutine. The subroutine is pre compiled, which do not have to compile, optimize, generate query plan for each call, to improve the query speed; event driven trigger is a special storage process. You can start another storage process through triggers, ensure database integrity; multi-threading is 
Sybase database manages the process without operating system, as a part of the connection between database to manage. The database engine also replace the operating system to manage the hardware resources, such as ports, memory and hard disk, which avoid the process operating system to improve the performance.

Sybase and SybaseSQLAnywhere are usually used in client / server environment. The former is used as the server database and the latter is the client database. It is widely used in large and medium scale systems. Sybase database system is a multibase structure, namely "Sybase Adaptive Server", which support and manage multiple database, including the database system, optional database and user database. The structure of the system as shown in Fig. 1 [4,5].

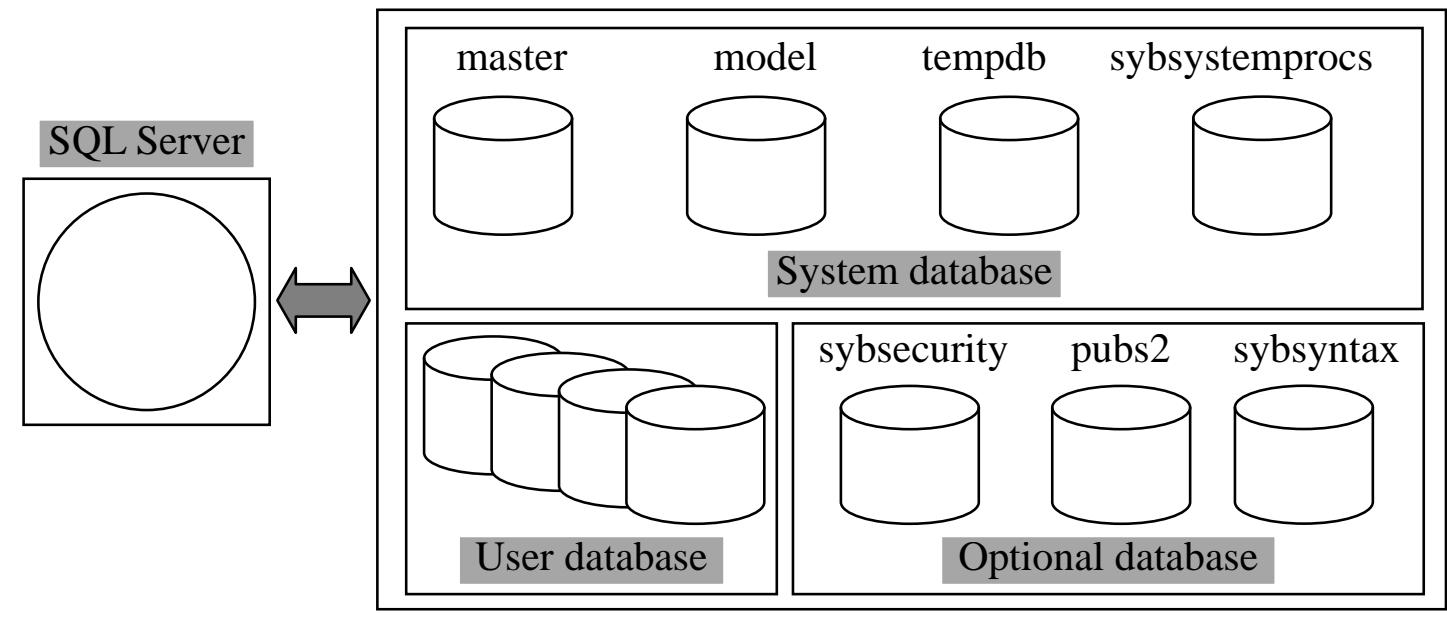

Fig. 1. Structure on Sybase database system

(1) System database [6]. Automatic generation of system database when installing Sybase. The master database, manage and control of a user database and maintain the normal operation of the server, save a lot of information systems, including the server configuration, user and equipment; the model database, in order to create a database user and provide the template. Whenever you create a new database, SQL server automatically build a model of copy and expanded to the size of user requirements, as a new user database; database tempdb is the temporary database, operation and processing server provides the shared memory region, to be shared by all users of all the database in the server; Database sybsystemprocs, used to preserve the system command. When the user database is running to SP at the beginning of the stored procedure, SQL server according to the following sequential search: the database, sybsystemprocs database, the master database.

(2) Optional database. Security audit database (sybsecurity), system security auditing information preservation, tracking record each user operation, to maintain system security control means; sample database pubs2), provide multiple learning Sybase database model for users; command syntax data library (sybsyntax), preserve Sybase database command syntax and language support library.

(3)The user database, is the real purpose of using the Sybase server. To manage user data, you must create a database in create, using the database Sybase command to create the database. Users who cannot access the master database have no right to create a new database.

\section{Conceptual Structure Design}

The concept structure is the common foundation of all kinds of data models, which is more independent in machine than the data model, more abstract, and thus more stable. Conceptual structure design is the key of the whole database design. The process of conceptual structure design is to get the demand analysis of the users, to abstract the process of the information structure, namely the process of the conceptual model. The conceptual structure design has the following characteristics: it can reflect the real world, it is easy to understand, easy to change, it is easy to change the data model, such as the relationship, gateway, level and so on. Abstract is a common 
method of conceptual structure. It is a common feature of the actual people, objects, things and concepts, and ignores the non-essential details, and describes them with all kinds of concepts. Course management system includes seven entities, namely "Course Type Dictionary, Teaching Type Dictionary, Examination Mode Dictionary, Teaching College Dictionary, Training Type Dictionary, Professional Dictionary, Course Comprehensive Information Table ", the seven entities exist six "one to many" relationship. The results of conceptual structure design are represented by E-R, as shown in Fig. 2 [7-10].

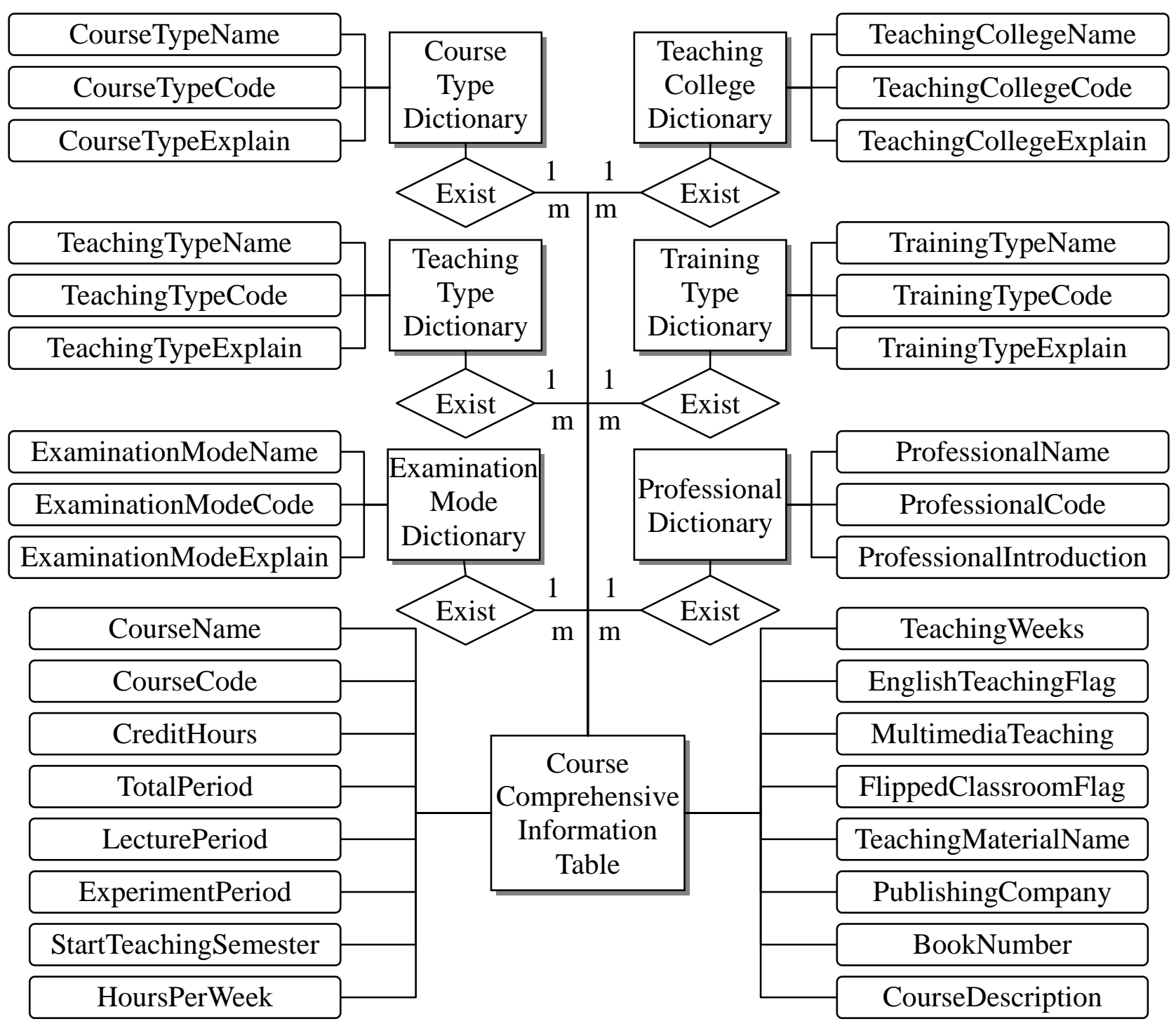

Fig. 2. Conceptual structure design on course management system

\section{Logical Structure Design}

Conceptual structure is the common foundation of all kinds of data models. In order to be able to use a certain DBMS to achieve user needs, it is necessary to transform the conceptual structure to the corresponding data model, which is the task of database logical structure design. The logical structure of the relationship model is a set of relational model. The E-R map into the relational model, is between the entities, attributes and entities linked into relation mode. A type is converted to a relational model, the E-R map contains 7 entities shown in Fig. 2, therefore, is converted into 7 mode, respectively, "CourseTypeDictionary, TeachingTypeDictionary, ExaminationModeDictionary, TeachingCollegeDictionary, TrainingTypeDictionary, ProfessionalDictionary, CourseComprehensiveInformationTable" A "to Many One" to convert to an independent relationship model, can also be combined with the "Many" side, the system uses the 
"Many" side of the merger. The logical structure of the design results are shown in Table 1.

Table 1. Logical structure design on course management system

\begin{tabular}{|c|c|c|c|c|c|c|c|}
\hline \multicolumn{4}{|c|}{ CourseTypeDictionary } & \multicolumn{4}{|c|}{ CourseComprehensiveInformationTable } \\
\hline No & Field name & Data type & Bytes & No & Field name & Data type & Bytes \\
\hline 11 & CourseTypeName & Varchar & 50 & 71 & CourseName & Varchar & 50 \\
\hline 12 & CourseTypeCode & Char & 4 & 72 & CourseCode & Char & 16 \\
\hline 13 & CourseTypeExplain & Text & & 73 & CourseTypeName & Varchar & 50 \\
\hline \multicolumn{4}{|c|}{ TeachingTypeDictionary } & 74 & CourseTypeCode & Char & 4 \\
\hline No & Field name & Data type & Bytes & 75 & TeachingTypeName & Varchar & 50 \\
\hline 21 & TeachingTypeName & Varchar & 50 & 76 & TeachingTypeCode & Char & 2 \\
\hline 22 & TeachingTypeCode & Char & 2 & 77 & ExaminationModeName & Varchar & 50 \\
\hline 23 & TeachingTypeExplain & Text & & 78 & ExaminationModeCode & Char & 1 \\
\hline \multicolumn{4}{|c|}{ ExaminationModeDictionary } & 79 & TeachingCollegeName & Varchar & 50 \\
\hline No & Field name & Data type & Bytes & 80 & TeachingCollegeCode & Char & 3 \\
\hline 31 & ExaminationModeName & Varchar & 50 & 81 & TrainingTypeName & Varchar & 50 \\
\hline 32 & ExaminationModeCode & Char & 1 & 82 & TrainingTypeCode & Char & 1 \\
\hline 33 & ExaminationModeExplain & Text & & 83 & ProfessionalName & Varchar & 50 \\
\hline \multicolumn{4}{|c|}{ TeachingCollegeDictionary } & 84 & ProfessionalCode & Char & 3 \\
\hline No & Field name & Data type & Bytes & 85 & CreditHours & Decimal & 4 \\
\hline 41 & TeachingCollegeName & Varchar & 50 & 86 & TotalPeriod & Tinyint & 1 \\
\hline 42 & TeachingCollegeCode & Char & 3 & 87 & LecturePeriod & Tinyint & 1 \\
\hline 43 & TeachingCollegeExplain & Text & & 88 & ExperimentPeriod & Tinyint & 1 \\
\hline \multicolumn{4}{|c|}{ TrainingTypeDictionary } & 89 & StartTeachingSemester & Tinyint & 1 \\
\hline No & Field name & Data type & Bytes & 90 & HoursPerWeek & Tinyint & 1 \\
\hline 51 & TrainingTypeName & Varchar & 50 & 91 & TeachingWeeks & Tinyint & 1 \\
\hline 52 & TrainingTypeCode & Char & 1 & 92 & EnglishTeachingFlag & Tinyint & 1 \\
\hline 53 & TrainingTypeExplain & Text & & 93 & MultimediaTeaching & Tinyint & 1 \\
\hline \multicolumn{4}{|c|}{ ProfessionalDictionary } & 94 & FlippedClassroomFlag & Tinyint & 1 \\
\hline No & Field name & Data type & Bytes & 95 & TeachingMaterialName & Varchar & 50 \\
\hline 61 & ProfessionalName & Varchar & 50 & 96 & PublishingCompany & Varchar & 50 \\
\hline 62 & ProfessionalCode & Char & 3 & 97 & BookNumber & Varchar & 20 \\
\hline 63 & ProfessionalIntroduction & Text & & 98 & CourseDescription & Text & \\
\hline
\end{tabular}

\section{Storage and Optimization Design}

(1) Storage space allocation. The data distribution in Sybase database is divided into the following layers according to the physical: equipment, segment, allocation unit, interval and page. In the process of creating the database, we first create a database device, and record the information of the device to the masters database in the sysdevices system table. In database Create, when a device is referenced, a portion of the device is allocated to a database segment. Allocation unit, interval, and page, each database is divided into several $0.5 \mathrm{~m}$ allocation units, each allocation unit is divided into 32 interval, an interval only with a database object association, namely database objects with the interval for the distribution; Each interval is divided into eight pages, pages are classified for data pages and index pages, distribution page, object allocation map page (OAM), global allocation map page (GAM). The table data are stored in The data pages, index page storage index entries, page allocation is record allocation unit in which interval has been in use, what pages to actual data, OAM page recorded an object with interval distribution page, GAM page record which allocation 
unit also has a range of object storage.

(2) Optimal design. Database design process optimization, refers to the index optimization and field design optimization, etc. Sybase index optimization is particularly important. Reasonable index optimization can improve the performance of the database, avoid the database bottleneck. In the database design, the database should be designed to make up less disk space. Some of the optimization techniques in the database design process are as follows:

$>$ As far as possible use the smaller the integer type (Tinying is more suitable than Int).

$>$ It is possible to define fields for the "NOT NULL", unless this field requires the "NULL".

$>$ If not used variable length field, such as "varchar", then use fixed size record format, such as "char".

$>$ The main index table is as short as possible, so that each record has the name logo and more efficient.

$>$ Only create the index really need. Index is conducive to the retrieval of records, but is not conducive to fast save records. If you always do a search on the combined field of a table, create an index on these fields. The first part of an index must be the most commonly used field. If you always need to use a lot of fields, you should first copy the fields and make the index better.

$>$ All data are processed in the stored database before.

$>$ All fields must have a default value.

\section{Conclusion}

Sybase is based on the client / server architecture of the database, to support the sharing of resources and balance the load between multiple devices, allowing the environment to accommodate a number of hosts, to take full advantage of a variety of existing enterprise systems. Between the client and the server, the network protocol is used to connect and communicate, the client sends a request to the server, the server responds the request, and carries on the corresponding service. The research results of this paper have solved the key technical problems of the system development, and laid the foundation for the rapid development of the application system.

\section{Acknowledgement}

This work is supported by 2013 annual social science planning fund project of Liaoning province (L13CTQ012): Research on investigation and cultivation for college students' reading tendency of digital resources; 2014 annual education science planning of Liaoning province (JG14DB019): Strategy research on teachers' teaching mode transformation and teaching ability in digital environment.

\section{References}

[1] X. Li, "The development and design of the university course management system," Electronic Technology \& Software Engineering, vol. 3, no. 21, pp. 92, 2014.

[2] Y. Yang, "School Curriculum Management System," Master's degree of Jilin University, 2012.

[3] 51CTO Technology Salon, "Introduction of SQL Server Sybase architecture," http://database.51cto.com/art/200902/104966.htm, 2016-3-10.

[4] W. J. Wu, B. Zhou, L. Shen, et al, "Maintenance Management of Sybase Database in Hospital Information System," China Medical Devices, vol. 30, no. 8, pp. 81-86, 2015.

[5] G. Z. Liu, X. L. Shi, "Analysis and Research on the architecture of Oracle and Sybase," http://wenku.baidu.com/link?url=anhPkh3YNMzFWtCxdxiw3JvHlK7ixsshF6EgZBed8z5AsY bghi8dEmfeHBmNc8g73HB3Guie6ZKGGz6C0wkeIDIPyFAhUZ1liOBgxpyWjAO, 2016-3-10. 
[6] IT expert network, "Composition and characteristics of Sybase database," http://database.ctocio.com.cn/172/12149172_2.shtml, 2016-3-12.

[7] P. F. Li, "Design and implementation of management system for comprehensive course in university based on.NET," Master's degree of Yanshan University, 2012.

[8] J. Li, "Design and Implementation of A Curriculum Information Management System for Universities and Colleges," Master's degree of Xiamen University, 2014.

[9] N. L. Zhang, "Development and Implementation of Process Management System of Experimental Course," Research and Exploration in Laboratory, vol. 33, no. 9, pp. 265-267, 2014.

[10] Z. H. Hu, F. Zhu, "The Design and Realization of Curriculum Management System of Training Departments Based on B/S Architecture," Computer Engineering \& Software, vol. 38, no. 8, pp. 79-83, 2015. 